\section{Dopamintransporter abnormal bei ADHS}

In früheren Studien stellte man Veränderungen in der Dichte des Dopamintransporters im Striatum von Patienten mit ADHS fest. Jedoch kamen Untersuchungen mit SPECT und PET zu widersprüchlichen Ergebnissen. Vor diesem Hintergrund führte ein Team von US-amerikanischen Wissenschaftlern eine Metaanalyse der zu diesem Thema erschienen Arbeiten durch.

$\mathrm{N}$ eun von 59 Studien mit 169 Patientendaten über die striatale Dopamintransporterdichte bei Erwachsenen erfüllten die Ausgangskriterien des Teams: 1. Veröffentlichung in einem „peer-reviewed“-Journal, 2. Kriterien für ADHS von DSMiV oder ICD 10 werden erfüllt, 3. analysierte Daten über die striatale Dopamintransporterdichte mit Hilfe von SPECT oder PET, 4. Standardabweichungen für die Dichte des Dopamintransporters werden angegeben und 5. Vergleich mit einer Kontrollgruppe.

Die Autoren stellten fest, dass bei Erwachsenen mit ADHS, die keine Stimulanzientherapie in ihrer Kindheit bzw. Jugend erhalten hatten, die Dopamintransporterdichte im Vergleich zur Kontrollgruppe erniedrigt war. Dagegen zeigten Erwachsene mit ADHS, denen eine Stimulanzienbehandlung in ihrer
Kindheit/Jugend verordnet worden war, eine erhöhte Dichte des Tranporters. Die Autoren schlussfolgern, dass bei einer ADHS primär eine erniedrigte Dopamintransporterdichte bestehe. Unter Medikation komme es zu einer kompensatorischen Erhöhung der Transporterdichte im Sinne einer Up-Regulation.

Fusar-Poli $P$ et al. Striatal dopamine transporter alterations in ADHD: pathophysiology or adaptation to psychostimulants? A meta-analysis. Am J Psychiatry 2012; 169: 264-72

Kommentar: Wieder einmal wird eine Arbeitshypothese - nämlich die, dass bei Menschen mit ADHS primär eine erhöhte striatale Dopamintransporterdichte bestehe, die durch Dopamintransporterblockade mittels Stimulanzien effektiv behandelt würde - auf den Prüfstein gestellt. Dies macht bewusst, dass wir bisher nur mit Hy- pothesen, aber nicht mit Gewissheiten arbeiten. Insbesondere wissen wir nicht, welches Glied in der pathophysiologischen Kette der Dopamintransporters darstellt. Weitere Studien werden erforderlich sein, um die vorliegende These zu erhärten oder zu widerlegen.

Insbesondere fehlen Informationen, inwieweit die untersuchten Erwachsenen, z.B. durch Nikotin, ihre Dopamintransporterdichte beeinflusst haben - es ist bekannt, dass Nikotin ebenfalls den Dopamintransporter blockiert und dass der Nikotinkonsum bei Erwachsenen mit ADHS erhöht ist. Auch ist es wichtig zu wissen, mit welchen Stimulanzien, wie lange und in welcher Dosis die vorbehandelten Erwachsenen therapiert wurden - und ob dies einen Einfluss auf die später gemessene Dopamintransporterdichte hatte. Sinkt im weiteren Verlauf ohne Stimulanzien die Dopamintransporterdichte wieder $\mathrm{ab}$ - und zwar stärker als physiologisch zu erwarten ist? Dafür sind Longitudinaluntersuchungen erforderlich. An dem Konzept, dass bei ADHS eine Störung im Bereich der neuronalen Transmission besteht mit einer Dysregulation von Dopamin-modulierten neuronalen Netzwerken, ändert sich jedoch mit den Ergebnissen dieser Studie bisher nichts.

Dr. Kirsten Stollhoff

\section{ADHS + Epilepsie: Unterscheidet sich die komorbide Form?}

Rund $30 \%$ der Kinder mit Epilepsie leiden an einer ADHS. Ärzte vom Universitätsspital Basel gingen nun der Frage nach, ob die genuine ADHS sich neuropsychologisch und funktionell unterscheidet von der ADHS, die komorbid mit Epilepsie auftritt.

D ie Wissenschaftler untersuchten 17 Jungen mit Epilepsie und ADHS (sechs mit Hyperaktivität, elf ohne Hyperaktivität), 15 Jungen mit genuiner ADHS (neun mit, sechs ohne Hyperaktivität) und eine Kontrollgruppe aus weiteren 15 Jungen mittels Testaufgaben, die eine Aktivierung des Arbeitsgedächtnisse erfordern. Es wurden jeweils zwei Testdurchläufe durchgeführt: mit und ohne Methylphenidat(MPH)-Medikation. Bei allen Testungen wurde gleichzeitig eine funktionelle Magnetresonanztomographie (fMRT) abgeleitet.
Die Autoren fanden in den Tests in beiden von ADHS betroffenen Gruppen nativ unterdurchschnittliche Leistungen im Vergleich zur Kontrollgruppe. Unter ner Normalisierung. Im fMRT wurde eine Unterfunktion der Bereiche der Netzwerke des Arbeitsgedächtnisse beobachtet - auch hier wieder identisch in den Gruppen mit ADHS mit bzw. ohne Epilepsie. In der Kontrollgruppe war dagegen eine Normfunktion dieser Netzwerke zu sehen. Unter der MPH-Medikation zeigen die fMRT-Bilder keine Veränderung, trotz klinischer „NormaMPH-Medikation kam es jedoch zu ei- lisierung" waren die funktionellen Bilder unverändert pathologisch.

Bechtel $\mathrm{N}$ et al. Attention-deficit/hyperactivity disorder in childhood epilepsy: A neuropsychological and functional imaging study. Epilepsia 2012; 53: 325-33

Kommentar: Die Untersuchungen der Autoren weisen darauf hin, dass identische Netzwerke sowohl bei ADHS als auch bei ADHS + Epilepsie betroffen sind. Dies spricht für eine beiden Störungen zugrunde liegende gemeinsame Pathophysiologie jedenfalls in Teilbereichen. Es wurden keine Unterschiede - weder testpsychologisch noch funktionell - gefunden zwischen Kindern mit ADHS mit Hyperaktivität und ohne Hyperaktivität. Erstaunlich ist, dass sich unter Medikation zwar das Arbeitsgedächtnis klinisch normalisiert, nicht aber in den bildgebenden Verfahren. Für die Praxis wichtig ist, dass Kinder mit Epilepsie und ADHS ebenfalls positiv auf eine MPH-Therapie ansprechen. Dr.Kirsten Stollhoff 